\section{Im Park übernachtet}

Nach einem Streit mit der Freundin wurde ein seit einem Tag vermisster 39-jähriger Mann vom Notarzt bei $-35^{\circ} \mathrm{C}$ auf einer Parkbank aufgefunden. Man bemerkte Alkoholgeruch in der Atemluft. Trotz dicker Winterkleidung und Winterstiefeln betrug die Körperkerntemperatur des Mannes nur noch $29^{\circ} \mathrm{C}$.

_ Im Krankenhaus begann man mit Reanimationsmaßnahmen. Zusätzlich erhielt der Mann erwärmte Infusionslösungen und wurde von außen angewärmt. Die Reanimation war erfolgreich. An Armen, Beinen, Händen und Füßen bemerkte man aber eine ausgeprägte Blasenbildung.
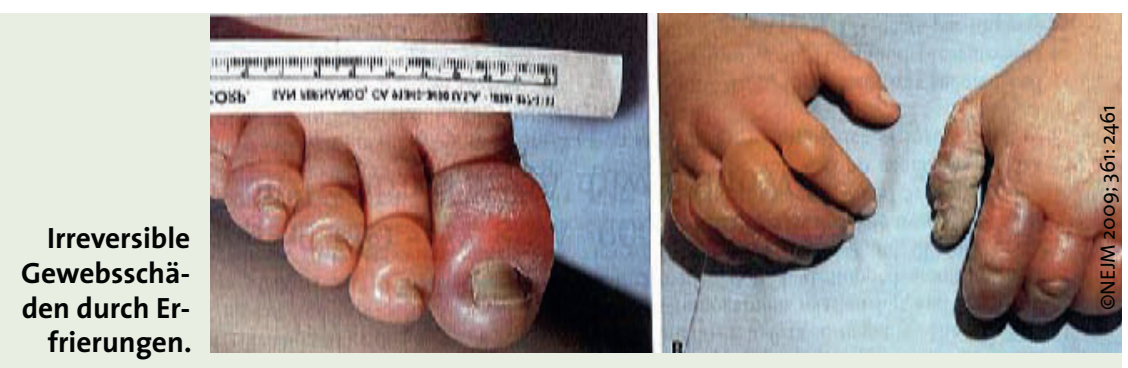

Die offensichtlichen Erfrierungen wurden täglich in einem warmen Bad behandelt und trocken verbunden, jedoch konnte man im Grunde nichts tun, als die Demarkierung der irreversiblen Gewebsschäden abzuwarten. Schließlich mussten die rechte Hand, der linke Mittelfinger und die linke Großzehe amputiert werden.

Kommentar: Das Risiko von Erfrierungen wird von vielen betroffenen Patienten nicht bedacht und falsch eingeschätzt.
Insbesondere bei gleichzeitiger Alkoholintoxikation und durchnässten Schuhen kann es bereits bei Temperaturen nur um den Gefrierpunkt zu schweren Erfrierungen kommen. Der initiale Lokalbefund lässt häufig auch noch nicht erahnen, welche katastrophale Entwicklung sich nach wenigen Tagen hier anbahnt.

H. S. FüeßI

Rehman H, Seguin A. Frostbite. New

Engl J Med 2009; 361: 2461

\title{
Chlamydien-Reinfektion im Blick
}

\begin{abstract}
Chlamydien sind gerade bei sexuell aktiven Adoleszenten ein häufiges Problem. Reinfektionen spielen hier eine wichtige Rolle, auch Therapieversager sind häufiger als erwartet. Wichtig ist neben der Behandlung auch die Information des Partners.
\end{abstract}

$\mathrm{D}$ ie Chlamydia-trachomatis-Infektion ist weltweit die häufigste sexuell übertragene bakterielle Erkrankung. Reinfektionen mit Chlamydien kommen häufig vor und sind für die hohe Zahl von akuten Infektionen verantwortlich. Sie sind oft Folge eines Nicht-Ansprechens der Antibiose, treten aber auch häufig nach ungeschütztem Sexualkontakt mit einem unbehandelten Partner bzw. einem infizierten neuen Partner auf.

In einer aktuellen Longitudinalstudie wurden 386 sexuell aktive adoleszente Mädchen im Alter von 14-17 Jahren alle drei Monate auf Chlamydia trachomatis untersucht. Der Nachweis erfolgte mittels Nukleinsäure-Amplifikationstest sowie durch Genotypisierung.

Insgesamt wurden 478 Episoden einer Chlamydia-trachomatis-Infektion bei 210 Mädchen nachgewiesen. 176 Mädchen blieben während des Studienzeitraums ohne eine Infektion. Die Inzidenz- rate betrug 34 Episoden/100 Frauenjahre. Von den infizierten Mädchen traten bei 121 wiederholte Infektion auf. Hier konnten 268 „Episodenpaare“ identifiziert werden. Von 183 Episodenpaaren lagen auswertbare Angaben vor: Bei $84,2 \%$ war eine Reinfektion gesichert, wahrscheinlich oder möglich. 13,7\% der Episoden wurden als Therapieversager beurteilt und 2,2\% galten als persistierend ohne eine dokumentierte Behandlung. Die Wirksamkeit der antibiotischen Therapie bei den jungen Frauen wurde mit 92,2\% berechnet. Die Autoren empfehlen ein Screeningprogramm auch bei Männern und ein verbessertes System für die Benachrichtigung der Partner.

Kommentar: Die Studie zeigt eindrucksvoll, dass Chlamydien-Reinfektionen bei hoher sexueller Aktivität häufig auftreten. Hier besteht Handlungsbedarf, um den bekannten Komplikationen vorzubeu- gen. Ein besseres System der Partnerbenachrichtigung könnte hier sicherlich helfen, sofern die Sexualpartner in einer Hochrisikogruppe (in der Reinfektionen eine entscheidende Rolle spielen) überhaupt bekannt sind. Problematisch ist auch die hohe Rate an Therapieversagern. Hier gilt es, die derzeitigen Therapiestandards zu überdenken und auch Kontrolluntersuchungen durchzuführen.

T.F. Schwarz

Batteiger BE et al. Repeated Chlamydia trachomatis genital infections in adolescent women. J Infect Dis 2010; 201: 42-52.

\section{Neuer Partner im Visier? Teenager, die sexuell aktiv sind, haben häufig mit Chlamydien zu kämpfen.}

